\title{
On tight contact structures with negative maximal twisting number on small Seifert manifolds
}

\author{
PAOLO GHIGGINI
}

\begin{abstract}
We study some properties of transverse contact structures on small Seifert manifolds, and we apply them to the classification of tight contact structures on a family of small Seifert manifolds.
\end{abstract}

57M50, 57R 17

\section{Introduction}

In this article $M\left(e_{0} ; r_{1}, r_{2}, r_{3}\right)$ with $e_{0} \in \mathbb{Z}$ and $r_{i} \in(0,1) \cap \mathbb{Q}$ will denote the 3 -manifold specified by the surgery diagram in Figure 1. It is well known that $M\left(e_{0} ; r_{1}, r_{2}, r_{3}\right)$ carries a Seifert fibration over $S^{2}$ with three singular fibres corresponding to the three small unknots in the surgery diagram. The manifolds belonging to this family will be called small Seifert manifolds.

The classification of tight contact structures on small Seifert manifolds has been the object of intense study in the last few years. The generic case, when $e_{0} \neq-1,-2$, was settled by $\mathrm{Wu}$ [20] and the author, Lisca and Stipsicz [5], who also studied a large family of manifolds with $e_{0}=-1$ in [6]. The goal of this article is the classification of tight contact structures on some small Seifert manifolds with $e_{0}=-2$. Such results are useful in symplectic cut-and-paste operations like the generalised symplectic rational blow-down; see Gay and Stipsicz [3].

The main invariant in the classification of tight contact structures on Seifert manifolds is the maximal twisting number. Let $L$ be a regular fibre for the Seifert fibration on $M=M\left(e_{0} ; r_{1}, r_{2}, r_{3}\right)$, and let $\mathcal{S}$ be the set of isotopies $\varphi:[0,1] \times M \rightarrow M$ such that $\varphi_{0}$ is the identity and $\varphi_{1}(L)$ is a Legendrian curve. $L$ has a distinct framing induced by the Seifert fibration, so we can transport this framing to $\varphi_{1}(L)$. We denote by $L_{\varphi}$ the framed curve $\varphi_{1}(L)$ with the framing induced by $\varphi$. As a Legendrian curve, $\varphi_{1}(L)$ has also a framing induced by the contact structure. We define the twisting number $\operatorname{tb}\left(L_{\varphi}\right)$ as the difference between the contact framing and the framing induced by $\varphi$. 


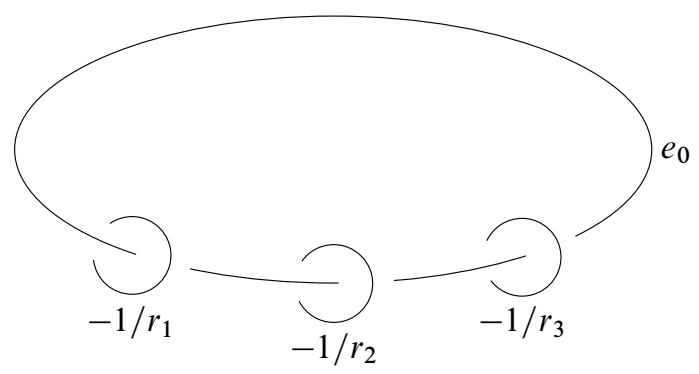

Figure 1: Rational surgery diagram for the Seifert fibred 3-manifold $M\left(e_{0} ; r_{1}, r_{2}, r_{3}\right)$

Definition 1.1 For any contact structure $\xi$ on $M$, we define the maximal twisting number of $\xi$ as

$$
t(\xi)=\max _{\varphi \in \mathcal{S}} \min \left\{t b\left(L_{\varphi}\right), 0\right\} .
$$

We can see $\mathcal{S}$ as the universal cover of the space of Legendrian curves isotopic to a regular fibre (vertical Legendrian curves from now on). However we would prefer to see the twisting number as a function on the space of vertical Legendrian curves, not on its universal cover. This is the case when the framing on $L_{\varphi}$ is independent of $\varphi$, and happens in the manifolds we are interested in. In fact if two isotopies induce different framings on the same vertical Legendrian curve, then the twisting number can be made arbitrarily big, so the contact structures has $t=0$. Moreover, if on a given manifold this happens for one tight contact structure, then it happens for all.

The first result of this article is a necessary and sufficient condition for the existence of tight contact structures with negative maximal twisting number on small Seifert manifolds with $e_{0}=-1$. Necessary and sufficient conditions for small Seifert manifolds with $e_{0} \neq-1$ were given by $\mathrm{Wu}$ [20]: tight contact structures with negative maximal twisting number exist on a small Seifert manifold with $e_{0} \neq-1$ if and only if $e_{0}$ is negative. Our condition is the following:

Theorem 1.2 Let $M\left(e_{0} ; r_{1}, r_{2}, r_{3}\right)$ be a small Seifert manifold with $e_{0}=-1$. Then the following facts are equivalent:

(1) $M\left(e_{0} ; r_{1}, r_{2}, r_{3}\right)$ carries a tight contact structure $\xi$ with $t(\xi)<0$.

(2) There exist integer numbers $p_{1}, p_{2}, p_{3}$, and $q$ with $q>0$ such that

- $\operatorname{gcd}\left(p_{i}, q\right)=1$ and $p_{i} / q<-r_{i}$,

- if $p^{\prime} / q^{\prime} \in\left(p_{i} / q,-r_{i}\right)$, then $q^{\prime}>q$.

(3) $M\left(e_{0}, r_{1}, r_{2}, r_{3}\right)$ carries a contact structure transverse to the Seifert fibration. 
For each of the three rational numbers $r_{1}, r_{2}, r_{3}$, we can write

$$
-1 / r_{i}=\left[a_{0}^{(i)}, a_{1}^{(i)}, \ldots, a_{m_{i}}^{(i)}\right]=a_{0}^{(i)}-\frac{1}{a_{1}^{(i)}-\frac{1}{\ddots-\frac{1}{a_{m_{i}}^{(i)}}}}, \quad i=1,2,3,
$$

for some uniquely determined integer coefficients

$$
\begin{gathered}
a_{0}^{(i)}, \cdots, a_{m_{i}}^{(i)} \leq-2, \quad i=1,2,3 . \\
T\left(r_{i}\right)=\prod_{k=0}^{m_{i}}\left|a_{k}^{(i)}+1\right| .
\end{gathered}
$$

We define

With the help of Theorem 1.2 we can classify tight contact structures on small Seifert manifolds with $e_{0}=-2$ which are $L$-spaces. An $L$-space was originally defined as a rational homology sphere $Y$ for which rk $\widehat{H F}(Y)=\left|H_{1}(Y, \mathbb{Z})\right|$. However, thanks to [16, Theorem 1.1], small Seifert manifolds $M$ with $e_{0}=-2$ which are $L$-spaces can be characterised as those for which $-M$ carries no contact structures transverse to the Seifert fibration.

Theorem 1.3 Let $M\left(-2 ; r_{1}, r_{2}, r_{3}\right)$ be an $L$-space. Then all tight contact structures on $M\left(-2 ; r_{1}, r_{2}, r_{3}\right)$ have maximal twisting number -1 and are Stein fillable. $M\left(-2 ; r_{1}, r_{2}, r_{3}\right)$ admits exactly $T\left(r_{1}\right) T\left(r_{2}\right) T\left(r_{3}\right)$ isotopy classes of tight contact structure, which are distinct by their $\operatorname{Spin}^{c}$-structures, and are filled by the Stein manifolds described by Legendrian surgery on all possible Legendrian realisations of the link in Figure 2.

Theorem 1.3 confirms the conjecture that, on small Seifert manifolds which are $L-$ spaces, all tight contact structure have nontrivial Ozsváth-Szabó invariant and are distinguished by the induced $\operatorname{Spin}^{c}$-structure [6, Conjecture 1.2].

Acknowledgements During the final preparation of this article the author was supported by CIRGET and by the Chaire de Recherche du Canada en algèbre, combinatoire et informatique mathématique de l'UQAM.

We warmly thank Paolo Lisca for sharing a preliminary version of his and Matić's work on transverse contact structures on Seifert manifolds [15], András Stipsicz for his encouragement and for suggesting the statement of Theorem 1.3, and $\mathrm{Hao} \mathrm{Wu}$ for sharing Figure 2. 


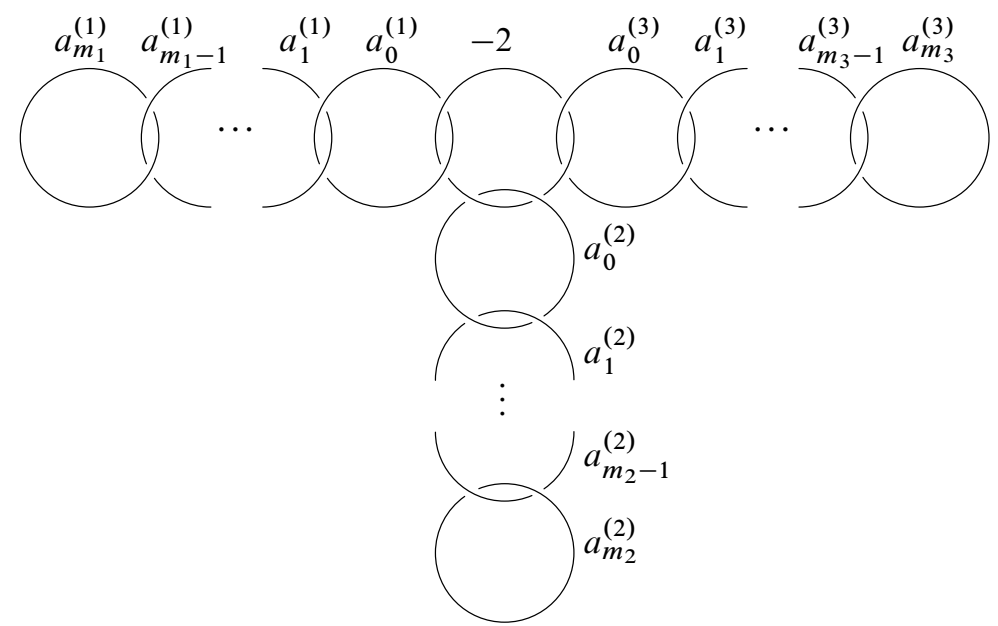

Figure 2: Integer surgery presentation of $M\left(-2 ; r_{1}, r_{2}, r_{3}\right)$

Some of the results in this paper have been independently obtained also by Patrick Massot [17]. We thank him for carefully reading the first version of this article and for suggesting several improvements.

\section{Decomposition of negative twisting contact manifolds}

In this section we prove constraints on the maximal twisting number, in particular proving a necessary condition for the existence of tight contact structures with negative maximal twisting number. The reader is assumed to be familiar with convex surfaces theory [7] and bypasses [11].

Let $V_{i}$ be a tubular neighbourhood of the singular fibre $F_{i}$ for $i=1,2,3$. We identify $-\partial\left(M \backslash V_{i}\right)$ with $\mathbb{R}^{2} / \mathbb{Z}^{2}$ so that $\left(\begin{array}{l}0 \\ 1\end{array}\right)$ is the direction of the regular fibres, and the meridian of $V_{i}$ has slope $-r_{i}$ in $-\partial\left(M \backslash V_{i}\right)$.

$M \backslash\left(V_{1} \cup V_{2} \cup V_{3}\right)$ is diffeomorphic to a product $\Sigma \times S^{1}$ where $\Sigma$ is a pair of pants, and sometime it will be useful to consider also a second set of coordinates on $-\partial\left(M \backslash V_{i}\right)$ coming from the product structure such that $\left(\begin{array}{l}1 \\ 0\end{array}\right)$ is the direction of the section $\Sigma \times\{1\}$. We choose the diffeomorphism between $M \backslash\left(V_{1} \cup V_{2} \cup V_{3}\right)$ and $\Sigma \times S^{1}$ so that boundary slopes $s_{1}, s_{2}$ and $s_{3}$ in the old bases will correspond to boundary slopes $s_{1}^{\prime}=s_{1}, s_{2}^{\prime}=s_{2}$, and $s_{3}^{\prime}=s_{3}-e_{0}$ in the bases coming from the product structure.

Proposition 2.1 Let $M=M\left(e_{0} ; r_{1}, r_{2}, r_{3}\right)$ be a small Seifert manifold with integer Euler class $e_{0}=-1$ or $e_{0}=-2$, and let $\xi$ be a tight contact structure on $M$ with 
maximal twisting number $t(\xi)=-q<0$. Then for $i=1,2,3$ there exist tubular neighbourhoods $U_{i}$ of the singular fibres $F_{i}$ such that $M \backslash\left(U_{1} \cup U_{2} \cup U_{3}\right)$ has minimal convex boundary with slopes $s\left(-\partial\left(M \backslash U_{i}\right)\right)=p_{i} / q$ with $q>0$ satisfying

(1) $\operatorname{gcd}\left(p_{i}, q\right)=1$ and $p_{i} / q<-r_{i}$,

(2) if $p^{\prime} / q^{\prime} \in\left(p_{i} / q,-r_{i}\right)$, then $q^{\prime}>q$.

Proof Let $L$ be a vertical Legendrian curve with twisting number $t b(L)=-q$. Isotope the Seifert fibration so that $L$ becomes a regular fibre and the singular fibres $F_{i}$ become Legendrian, then take standard neighbourhoods $V_{i}$ of $F_{i}$. We can make the twisting numbers of $F_{i}$ negative and as big as we wish in absolute value, therefore making the slopes of $-\partial\left(M \backslash V_{i}\right)$ arbitrarily close to $-r_{i}$. By repeatedly attaching the bypasses coming from convex vertical annuli with Legendrian boundary between $L$ and $-\partial\left(M \backslash V_{i}\right)$ (Imbalance Principle [11, Proposition 3.17]), we obtain tubular neighbourhoods $U_{i}$ of $F_{i}$ containing $V_{i}$ such that $-\partial\left(M \backslash U_{i}\right)$ has slope $p_{i} / q$. The numbers $p_{i} / q$ are uniquely determined by being the first ones which have denominator not greater than $q$ in the shortest path in the Farey tessellation from the slope of $-\partial\left(M \backslash V_{i}\right)$ to infinity. In particular, if $t(\xi)=-1$, then $q=1, p_{i} / q=\left[-r_{i}\right]$, and the properties of $p_{i} / q$ follow immediately.

Assume now $t(\xi)<-1$, so that $q>1$. The property $\operatorname{gcd}\left(p_{i}, q\right)=1$ follows from $t(\xi)=-q$ because, if the fraction $p_{i} / q$ could be reduced for some $i$, then the twisting number of a vertical Legendrian ruling curve of $-\partial\left(M \backslash U_{i}\right)$ would be greater than $-q$. Since the vertical Legendrian ruling curves of $-\partial\left(M \backslash U_{i}\right)$ are smoothly isotopic to regular fibres, this would contradict $t(\xi)=-q$. Since $-\partial\left(M \backslash U_{i}\right)$ is obtained from $-\partial\left(M \backslash V_{i}\right)$ by attaching vertical bypasses, and the attachment of a vertical bypasses decreases the slope, we have $p_{i} / q<-r_{i}$ for $i=1,2$.

We prove point 2 by contradiction. Assume there is a rational number $p^{\prime} / q^{\prime} \in$ $\left(p_{i} / q,-r_{i}\right)$ with $q^{\prime} \leq q$ for some $i$. If $q^{\prime}=q$, then $\left(p_{i}+1\right) / q \in\left(p_{i} / q,-r_{i}\right)$. By the following algebraic Lemma 2.2, there is a fraction $p^{\prime} /(q-1) \in\left(p_{i} / q,\left(p_{i}+1\right) / q\right]$, therefore we can assume $q^{\prime}<q$.

By [2, Lemma 2.15] there is a neighbourhood $U_{i}^{\prime}$ of the singular fibre $F_{i}$ such that $-\partial\left(M \backslash U_{i}^{\prime}\right)$ has slope $p^{\prime} / q^{\prime}$. If we put $-\partial\left(M \backslash U_{i}^{\prime}\right)$ in standard form, a vertical Legendrian ruling curve will be a Legendrian curve with twisting number $-q^{\prime}>-q$ smoothly isotopic to a regular fibre. This contradicts the hypothesis $t(\xi)=-q$.

Lemma 2.2 For any rational number represented by a fraction $p / q$ with $q>1$ there exists an integer number $p^{\prime}$ such that $p^{\prime} /(q-1) \in[p / q,(p+1) / q]$. Moreover, if $p / q$ is a reduced fraction, then $p^{\prime} /(q-1) \neq p / q$. 
Proof Consider $n=[p / q]$ and divide the interval $[n, n+1]$ into $q$ subintervals $[i / q,(i+1) / q]$ for $i=n q, \ldots,(n+1) q$. The $q$ numbers $j /(q-1)$ for $j=$ $n(q-1), \ldots,(n+1)(q-1)$ must divide among the $q$ subintervals, and there can be at most one in each interval because $1 / q<1 /(q-1)$.

Definition 2.3 We will call $\left(M \backslash\left(U_{1} \cup U_{2} \cup U_{3}\right),\left.\xi\right|_{M \backslash\left(U_{1} \cup U_{2} \cup U_{3}\right)}\right)$ the background of $(M, \xi)$.

The possible backgrounds will be studied in the next section.

Lemma 2.4 Let $\xi$ be a tight contact structure with $t(\xi)<0$ on a Seifert manifold $M\left(e_{0} ; r_{1}, r_{2}, r_{3}\right)$ with integer Euler class $e_{0}=-1$. Then $t(\xi)<-1$.

Proof Assume by contradiction that $t(\xi)=-1$. By Proposition $2.1 p_{i} / q=-1$. We would like to apply the classification theorem [12, Lemma 5.1] to the background of $(M, \xi)$. However Honda orients the boundary by the outward normal convention, and uses the bases coming from the product structure, therefore with his conventions the boundary slopes of the background become 1, 1, and 0. By [12, Lemma 5.1 (3a)], the background of $(M, \xi)$ has a vertical Legendrian curve $L$ with $t b(L)=0$, contradicting the hypothesis $t(\xi)<0$.

The next lemma is a technical observation which will be repeatedly useful in the paper.

Lemma 2.5 Let $L$ be a maximally twisting Legendrian curve. If $A$ is a convex annulus with Legendrian boundary and one of its boundary components coincides with $L$, then the dividing set of $A$ contains no arcs with both endpoints on $L$.

Proof A dividing curve on $A$ with both endpoints on $L$ gives a bypass attached to $L$ as explained in Honda [11, Proposition 3.17]. It is well known that the attachment of a bypass decreases the twisting number (see Etnyre [1, Lemma 2.20]) contradicting our assumption.

Proposition 2.6 Let $M=M\left(e_{0} ; r_{1}, r_{2}, r_{3}\right)$ be a small Seifert manifold with integer Euler class $e_{0}=-1$ or $e_{0}=-2$, and let $\xi$ be a tight contact structure on $M$ with maximal twisting number $t(\xi)=-q<0$. If $p_{1} / q, p_{2} / q$, and $p_{3} / q$ are the boundary slopes of the background of $(M, \xi)$, then $p_{1}+p_{2}+p_{3}=e_{0} q-1$. 
Proof If $t(\xi)=-1$, then $e_{0}=-2$ by Lemma 2.4. In this case Proposition 2.1 implies $p_{1}=p_{2}=p_{3}=-1$, so the equality holds.

Assume now $t(\xi)=-q<-1$. Let $A$ be a convex vertical annulus between $-\partial\left(M \backslash U_{1}\right)$ and $-\partial\left(M \backslash U_{2}\right)$. The dividing set of $A$ has no boundary parallel dividing curves by Lemma 2.5 because $-q$ is the maximal twisting number.

By the edge-rounding lemma [11, Lemma 3.11], a neighbourhood of $U_{1} \cup U_{2} \cup A$ has boundary slope $\left(p_{1}+p_{2}+1\right) / q$. A complement of this neighbourhood is a tubular neighbourhood $U_{3}^{\prime}$ of $F_{3}$ containing $U_{3}$ such that $-\partial\left(M \backslash U_{3}^{\prime}\right)$ has slope $-\left(p_{1}+p_{2}+1\right) / q+e_{0}=-\left(p_{1}+p_{2}+1-q e_{0}\right) / q$. If $-\left(p_{1}+p_{2}+1-q e_{0}\right) / q \neq p_{3} / q$, then there would be a rational number $p^{\prime} /(q-1) \in\left[p_{3} / q,-\left(p_{1}+p_{2}+1-q e_{0}\right) / q\right]$ by Lemma 2.2. Then by [11, Proposition 4.16] there would be a convex torus parallel to $-\partial\left(M \backslash U_{3}^{\prime}\right)$ in $U_{3}^{\prime} \backslash U_{3}$ with slope $p^{\prime} /(q-1)$ computed with respect to the basis of $-\partial\left(M \backslash U_{3}\right)$. This would contradict $t(\xi)=-q$.

Corollary 2.7 Let $\xi$ be a tight contact structure with $t(\xi)<-1$ on a small Seifert manifold $M=M\left(e_{0} ; r_{1}, r_{2}, r_{3}\right)$ with integer Euler class $e_{0}=-2$. Then $t(\xi) \leq-4$.

Proof Let $p_{1} / q, p_{2} / q$, and $p_{3} / q$ be the boundary slopes of the background of $(M, \xi)$ as in Proposition 2.1. Recall that Proposition 2.1(2) implies $-1<p_{i} / q$ (which is equivalent to $-q-p_{i} \leq-1$ ) when $q>1$. From Proposition 2.6 we get $p_{1}=$ $-p_{2}-p_{3}-2 q-1$, therefore $p_{1} \leq-3$. This implies $q>3$ because $p_{1} / q>-1$.

\section{Tight contact structures on $\Sigma \times S^{1}$}

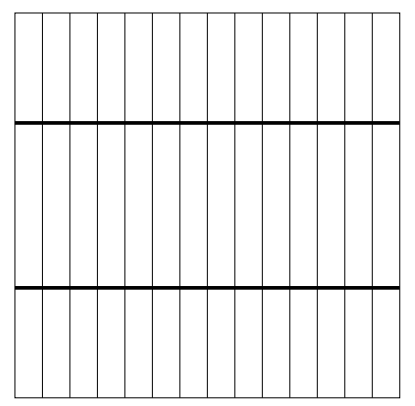

Figure 3: The standard characteristic foliation $\mathcal{F}(0,1)$ on $T^{2}$

In this section we classify all possible backgrounds. Given a pair of integer numbers $(p, q)$ with $q>0$, we denote by $\mathcal{F}(p, q)$ the standard characteristic foliation on $T^{2}$ with vertical Legendrian ruling, slope $p / q$ and $2 \operatorname{gcd}(p, q)$ Legendrian divides. Figure 3 shows $\mathcal{F}(0,1)$. 
Lemma 3.1 Let $\Sigma$ be a pair of pants, and consider coordinates on $-\partial\left(\Sigma \times S^{1}\right)=$ $T_{1} \cup T_{2} \cup T_{3}$ coming from the product structure. For every triple of integer numbers $\left(p_{1}, p_{2}, q\right)$ with $q>0$ there exists a contact structure $\beta\left(p_{1}, p_{2}, q\right)$ on $\Sigma \times S^{1}$ which is tangent to the fibres, and induces the characteristic foliations $\mathcal{F}\left(p_{1}, q\right), \mathcal{F}\left(p_{2}, q\right)$, and $\mathcal{F}\left(p_{3}, q\right)$ with $p_{3}=-\left(p_{1}+p_{2}+1\right)$ on the three components of the boundary of $\Sigma \times S^{1}$.

Proof Let $T_{1} \times[0,1]$ and $T_{2} \times[0,1]$ be invariant neighbourhoods of standard tori with characteristic foliations $\mathcal{F}\left(p_{1}, q\right)$ and $\mathcal{F}\left(p_{2}, q\right)$ respectively. Inside $\mathbb{R}^{2} \times S^{1}$ with the contact structure defined by the 1 -form $\cos (2 \pi q z) d x+\sin (2 \pi q z) d y$ we take the subset $A \times S^{1}$, where $A$ is the subset of the plane portrayed in Figure 4. If we glue $T_{1} \times[0,1], T_{2} \times[0,1]$, and $A \times S^{1}$ as in the picture, we obtain $\Sigma \times S^{1}$ with the contact structure $\beta\left(p_{1}, p_{2}, q\right)$.

Lemma 3.2 $\beta\left(p_{1}, p_{2}, q\right)$ is tight and its maximal twisting number is $-q$.

Proof Suppose there is an overtwisted disc or a vertical Legendrian curve with twisting number greater than $-q$. Then there is a convex annulus $B^{\prime}$ with Legendrian boundary which is smoothly isotopic to the annulus $B$ shown in Figure 4 and is disjoint from the overtwisted disc or the Legendrian curve. By the Isotopy Discretisation Lemma $\left[13\right.$, Lemma 3.10 ${ }^{1}$ there is a sequence of smoothly isotopic annuli $B=B_{0}, \ldots, B_{n}=$ $B^{\prime}$, all with the same boundary, such that for each $i$ the interior parts of $B_{i-1}$ and $B_{i}$ are disjoint, and $B_{i}$ is obtained from $B_{i-1}$ by the attachment of a single bypass.

We will prove that all annuli $B_{i}$, and in particular $B_{n}=B^{\prime}$, satisfy the following properties after a $C^{0}$-small modification:

(1) $B_{i}$ is contact isotopic to $B_{0}$.

(2) If we cut $\Sigma \times S^{1}$ along $B_{i}$ and round the edges we obtain two connected components isomorphic to $T_{1} \times[0,1]$ and $T_{2} \times[0,1]$ with the invariant contact structures inducing the characteristic foliation $\mathcal{F}\left(p_{1}, q\right)$ on the boundary components of $T_{1} \times[0,1]$, and $\mathcal{F}\left(p_{2}, q\right)$ on the boundary components of $T_{2} \times[0,1]$.

This will prove the Lemma because in the invariant contact structures on $T_{1} \times[0,1]$ and $T_{2} \times[0,1]$ there are neither overtwisted discs, nor vertical Legendrian curves with twisting number greater than $-q$.

\footnotetext{
${ }^{1}$ The proof in the reference is incomplete because it assumes that nonconvex surfaces form a discrete set in any generic contact film. This is not true, however any contact film starting and ending with a convex surface can be isotoped relative to the boundary to a contact film with such property by [10, Lemma 15].
} 
Properties (1) and (2) hold for $B=B_{0}$ by construction. In order to prove that they hold for $B_{i}$, we assume that they hold for $B_{i-1}$, and prove that $B_{i}$ is contact isotopic to $B_{i-1}$. Since $B_{i}$ is disjoint from $B_{i-1}$ outside the boundary, it is contained in one of the two connected components of $\Sigma \times S^{1} \backslash B_{i-1}$ (say in the one isomorphic to $T_{1} \times[0,1]$ to fix the notation). The bypass sending $B_{i-1}$ to $B_{i}$ can be trivial, can change the slope of $T_{1} \times\{1\}$ if $\operatorname{gcd}\left(p_{1}, q\right)=1$, or can decrease the number of dividing curves of $T_{1} \times\{1\}$ if $\operatorname{gcd}\left(p_{1}, q\right)>1$. The last two options are impossible because the contact structure on $T_{1} \times[0,1]$ is invariant. If the bypass sending $B_{i-1}$ to $B_{i}$ is trivial, then the dividing set of $B_{i}$ is isotopic to the dividing set of $B_{i-1}$, therefore we can modify $B_{i}$ in a small $C^{0}$ neighbourhood so that its characteristic foliation becomes isotopic to the characteristic foliation of $B_{i-1}$. After this modification $B_{i-1}$ is contact isotopic to $B_{i}$ and $\Sigma \times S^{1} \backslash B_{i}$ is contact isotopic to $\Sigma \times S^{1} \backslash B_{i-1}$ by the triviality of the attachment of trivial bypasses [13, Lemma 2.10]. The modification of the characteristic foliation of $B_{i}$ is implicitly required by [13, Lemma 2.10].

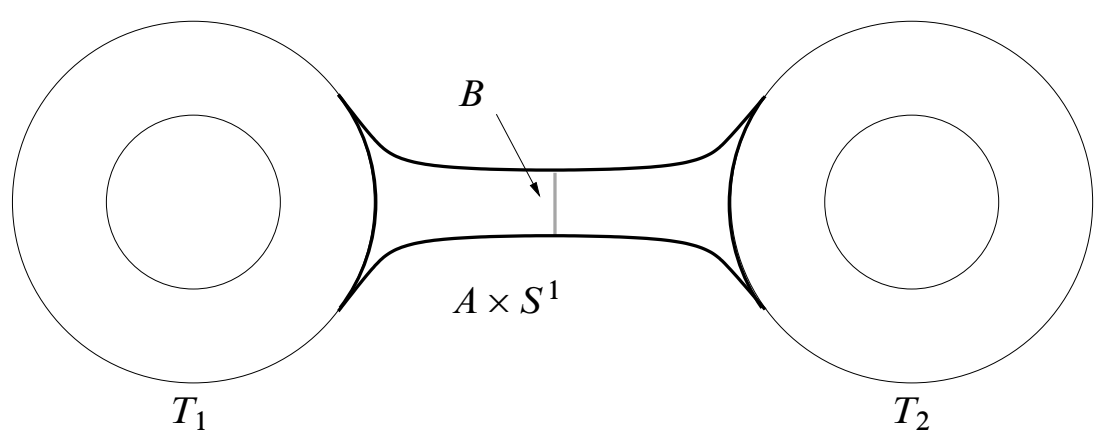

Figure 4: The construction of $\beta\left(p_{1}, p_{2}, q\right)$

Proposition 3.3 Up to an isotopy not necessarily fixed on the boundary, $\beta\left(p_{1}, p_{2}, q\right)$ is the unique tight contact structure on $\Sigma \times S^{1}$ with maximal twisting number $-q$ inducing the characteristic foliations $\mathcal{F}\left(p_{1}, q\right), \mathcal{F}\left(p_{2}, q\right)$ and $\mathcal{F}\left(p_{3}, q\right)$ with $p_{3}=$ $-\left(p_{1}+p_{2}+1\right)$ on the boundary.

Proof Take a convex annulus $A$ in $\left(\Sigma \times S^{1}, \xi\right)$ with Legendrian boundary between two vertical Legendrian ruling curves of $T_{1}$ and $T_{2}$ with twisting number $-q$. The dividing set of $A$ consists of curves with endpoints on different boundary components because of Lemma 2.5 and the maximality of the twisting number of $\partial A$. After an isotopy of $\beta\left(p_{1}, p_{2}, q\right)$ not fixed on the boundary, we can assume that the dividing set of $A$ consists of $2 q$ horizontal curves. After cutting along $A$ and rounding the edges by [11, Edge Rounding] we obtain a toric annulus $N$ diffeomorphic to $T_{3} \times[0,1]$ with 
characteristic foliation $\mathcal{F}\left(p_{3}, q\right)$ on both boundary components. The contact structure $\left.\beta\left(p_{1}, p_{2}, q\right)\right)$ restricted to $N$ is nonrotative, otherwise there would be a curve isotopic to a fibre with twisting number 0 , contradicting $t\left(\beta\left(p_{1}, p_{2}, q\right)\right)=-q$. For the same reason there can be no intermediate convex torus with fewer dividing curves.

Put the characteristic foliation on the boundary of $N$ in standard form so that each Legendrian ruling curve intersects each dividing curve exactly once. The considerations above imply that a Legendrian ruling curve of $\partial N$ minimises the twisting number in its isotopy class in $N$. Let $B$ be a convex annulus between two Legendrian ruling curves in different boundary components of $N$. By [11, Theorem 2.2(4)] its dividing set determines the isotopy class of the contact structure on $N$. The dividing set of $B$ contains no boundary parallel dividing arcs because of Lemma 2.5 , therefore it can be made horizontal with an isotopy of $N$ not fixed on the boundary, therefore the contact structure on $N$ is isotopic to the invariant contact structure by the same isotopy. This implies that $\beta\left(p_{1}, p_{2}, q\right)$ is unique up to isotopy not fixed on the boundary because it is determined by its restriction to the complement of a neighbourhood of $A$, and by the dividing set of $A$.

Since a contact isotopy of the background can be extended to a contact isotopy of $(M, \xi)$ even if it is not constant on the boundary [4, Lemma 4.4], we have the following corollary.

Corollary $3.4(M, \xi)$ has a background which is isotopic to $\beta\left(p_{1}, p_{2}, q\right)$ with $q=$ $-t(\xi)$.

\section{Transverse contact structures}

In the following a transverse contact structure on a Seifert manifold will be a contact structure which is positively transverse to the Seifert fibration. This condition has strong consequences both on the contact structure and on the topology of the underlying manifold. For example:

Theorem 4.1 ([15, Corollary 2.2]) Transverse contact structures on Seifert manifolds are weakly symplectically fillable and universally tight.

Proposition 4.2 If $\xi$ is a transverse contact structure on a small Seifert manifold $M$, then $t(\xi)<0$. 
Proof The base space of a small Seifert manifold is a 2-dimensional orbifold with three cone points having $S^{2}$ as underlying surface. The three cone points are the images of the singular fibres. Any such orbifold is finitely covered in the sense of orbifolds by a smooth surfaces $\Sigma^{\prime}$ (see Scott [19]) therefore $\pi_{1}\left(\Sigma^{\prime}\right)<\pi_{1}^{\text {orb }}(\Sigma)$ is a finite index subgroup. The fundamental group of $M$ fits into an exact sequence

$$
1 \longrightarrow K \longrightarrow \pi_{1}(M) \longrightarrow \pi_{1}^{\text {orb }}(\Sigma) \longrightarrow 1
$$

where $K$ is a cyclic group generated by the homotopy class of a regular fibre. The preimage of $\pi_{1}\left(\Sigma^{\prime}\right)$ in $\pi_{1}(M)$ is a finite index subgroup. Associated to this subgroup there is a finite cover $M^{\prime} \rightarrow M$. The pull back of the Seifert fibration to $M^{\prime}$ is a honest circle bundle over $\Sigma^{\prime}$ because the singular set of $\Sigma^{\prime}$ is empty.

If $\xi$ is transverse to the Seifert fibration of $M$, the pulled back contact structure $\xi^{\prime}$ is transverse to the fibres of $M^{\prime}$. By [9, Theorem 2.3], a universally tight contact structure on a circle bundle over a surface is transverse if and only if there is no Legendrian curve with twisting number 0 isotopic to a fibre.

Remark A second proof of this lemma can be given by applying the slice ThurstonBennequin inequality of Kronheimer and Mrowka to the regular fibres in the symplectic fillings constructed by Lisca and Matić in the proof of [15, Theorem 2.2].

Lemma 4.3 For every triple of integer numbers $\left(p_{1}, p_{2}, q\right)$ with $q>0$ we can perturb $\beta\left(p_{1}, p_{2}, q\right)$ in any arbitrarily small $C^{\infty}$-neighbourhood, and obtain a new contact structure $\widetilde{\beta}\left(p_{1}, p_{2}, q\right)$ on $\Sigma \times S^{1}$ which is transverse to the $S^{1}$-fibres and has convex boundary with the same dividing set as $\beta\left(p_{1}, p_{2}, q\right)$.

Proof Fix a contact form $\alpha$ for $\beta\left(p_{1}, p_{2}, q\right)$. Let $d z$ be the pullback of a volume form on $S^{1}$ to $\Sigma \times S^{1}$, and $\frac{\partial}{\partial z}$ be a vector field tangent to the $S^{1}$-fibration such that $d z\left(\frac{\partial}{\partial z}\right)=1$. Then, for $\epsilon$ small, the 1 -form $\alpha+\epsilon d z$ defines a contact structure which is transverse to the fibration because $\alpha\left(\frac{\partial}{\partial z}\right)=0$.

Put coordinates $(x, y, z)$ near a boundary component of $\Sigma \times S^{1}$, so that $z$ is the direction of the fibres and $x$ is the direction of the inward normal. The contact structure in a neighbourhood of a boundary component with characteristic foliation $\mathcal{F}(p, q)-$ the type of characteristic foliation induced by $\beta$ on the boundary of $\Sigma \times S^{1}-$ is locally defined by the 1 -form $\alpha=\cos (2 \pi(q z+p y)) d x+\sin (2 \pi(q z+p y)) d y$, and $\frac{\partial}{\partial x}$ is a contact vector field. It is straightforward to check that $\frac{\partial}{\partial x}$ is a contact vector field for the kernel of $\alpha+\epsilon d z$ too, and that the dividing set remains unchanged. 
Lemma 4.4 Let $\mathcal{F}_{0}$ and $\mathcal{F}_{1}$ be foliations on $T^{2}$ divided by the same multicurve $\Gamma$, and which are both transverse to the same choice of a vertical direction on $T^{2}$. Then there exists a transverse contact structure on $T^{2} \times I$ which induces $\mathcal{F}_{0}$ on $T^{2} \times\{0\}$ and $\mathcal{F}_{1}$ on $T^{2} \times\{1\}$ as characteristic foliations.

Proof Let $\omega$ be an area form on $T^{2}$. In the proof of [7, Proposition II.3.6], Giroux constructs a family of vector fields $Y_{s}=(1-s) Y_{0}+s Y_{1}$ and functions $v_{s}: T^{2} \rightarrow \mathbb{R}$ such that $Y_{i}$ directs $\mathcal{F}_{i}$ for $i=0,1$, and $\iota_{Y_{s}} \omega+v_{s} d t$ is a contact form on $T^{2} \times \mathbb{R}$ for all $s \in[0,1]$. By [8, Lemma 2.3] there is a function $u: T^{2} \times \mathbb{R} \rightarrow \mathbb{R}$ such that ${ }^{\iota} Y_{t} \omega+u_{t} d t$ is a contact form on $T^{2} \times[0,1]$. The transversality condition on $\mathcal{F}_{0}$ and $\mathcal{F}_{1}$ is equivalent to the existence of a 1 -form $\lambda$ on $T^{2}$ such that $\lambda\left(Y_{i}\right)>0$ for $i=0,1$. Since $\lambda\left(Y_{t}\right)>0$ for any $t \in[0,1]$ by linearity, the contact structure defined by $\iota_{Y_{t}} \omega+u_{t} d t$ is transverse.

Theorem 4.5 A small Seifert manifold $M=M\left(e_{0} ; r_{1}, r_{2}, r_{3}\right)$ with $e_{0}=-2,-1$ admits a positively transverse contact structure if and only if there are integer numbers $p_{1}, p_{2}, p_{3}$ and $q>0$ such that

(1) $p_{i} / q<-r_{i}$, and

(2) $p_{1}+p_{2}+p_{3}=q e_{0}-1$.

Remark Different, but equivalent, necessary and sufficient conditions for the existence of transverse contact structures on small Seifert manifolds have been proved by Lisca and Matić using 4-dimensional techniques and pre-existing results on taut foliations on small Seifert manifolds [15].

Proof The "only if" part follows from Proposition 2.1, Proposition 2.6 and Proposition 4.2. For the "if" part take neighbourhoods $U_{1}, U_{2}$, and $U_{3}$ of the singular fibres as in Proposition 2.1, and for $i=1,2,3$ denote by $s_{i}$ the boundary slope of $U_{i}$ corresponding to $p_{i} / q$ in the basis of $-\partial\left(M \backslash U_{i}\right)$. Let $\xi_{i}$ be the contact structure on $U_{i} \cong D^{2} \times S^{1}$ with coordinates $(\rho, \phi, \theta)$ defined by the equation $\xi_{i}=\operatorname{ker}\left(\cos \left(k_{i} \rho\right) d \theta+\right.$ $\left.k_{i} \rho \sin \left(k_{i} \rho\right) d \phi\right)$, where $k_{i}$ has been chosen so that $\partial U_{i}$ is pre-Lagrangian and has slope $s_{i}$. For $i=1,2,3, \xi_{i}$ is transverse to the Seifert fibration of $M$ restricted to $U_{i}$. In fact, by the invariance of $\xi_{i}$ in the directions $\theta$ and $\phi$, a tangency between $\xi_{i}$ and a regular fibre would produce a pre-Lagrangian torus where the leaves of the characteristic foliation coincides with the fibres of the Seifert fibration. A convex perturbation of this torus (as constructed in [4, Lemma 3.4]) would have infinite slope in the basis of $-\partial\left(M \backslash U_{i}\right)$. This is impossible because the slopes in $U_{i}$, computed in the basis of $-\partial\left(M \backslash U_{i}\right)$, belong to the interval $\left[p_{i} / q,-r\right)$. 
The boundary of $U_{i}$ can be made convex with $2 \operatorname{gcd}\left(p_{i}, q\right)$ dividing curves by a $C^{\infty}$-small perturbation of $\xi_{i}$, which therefore does not affect transversality; see [4, Lemma 3.4]. Then for $i=1,2,3$ we use Lemma 4.4 to make the characteristic foliations of $\widetilde{\beta}\left(p_{1}, p_{2}, q\right)$ on $-\partial\left(M \backslash U_{i}\right)$ and the characteristic foliation of $\xi_{i}$ on $\partial U_{i}$ match under the gluing maps, still without affecting transversality. When we glue all pieces together, we get a contact structure on $M$ which is always transverse to the Seifert fibration.

Remark If $e_{0}=-2$ we can always take $p_{1}=p_{2}=p_{3}=-1$ and $q=1$, so every small Seifert manifold with $e_{0}=-2$ admits a transverse tight contact structure.

Corollary 4.6 A small Seifert manifold $M\left(e_{0} ; r_{1}, r_{2}, r_{3}\right)$ admits a tight contact structure $\xi$ with $t(\xi)<0$ if and only if it admits a transverse contact structure.

Proof When $e_{0} \neq-1$ it follows from works of Wu [20] and Lisca and Matić [15] combined. When $e_{0}=-1$ one direction has been proved in Proposition 4.2. To prove the other direction assume that $M\left(e_{0} ; r_{1}, r_{2}, r_{3}\right)$ admits a tight contact structure with negative twisting. Then, combining Proposition 2.1(1) with Proposition 2.6, we obtain integer number $p_{1}, p_{2}, p_{3}$, and $q>0$ such that $\operatorname{gcd}\left(p_{1}, q\right)=1, p_{i} / q<-r_{i}$, and $p_{1}+p_{2}+p_{3}=q e_{0}-1$, therefore $M\left(e_{0} ; r_{1}, r_{2}, r_{3}\right)$ admits a transverse contact structure by Theorem 4.5 .

Proof of Theorem 1.2 The theorem follows from Proposition 2.1, Proposition 4.2 and Theorem 4.5.

\section{Tight contact structures on $L$-spaces}

In this section we classify tight contact structures on those small Seifert manifolds with $e_{0}=-2$ which are $L$-spaces. The condition of being an $L$-space is used to give a bound on the maximal twisted number, which in turn gives an upper bound on the number of tight contact structures. Finally we construct enough distinct tight contact structures to match the upper bound. After the bound on the maximal twisted number (Proposition 5.1) the proof goes on like in [20].

Proposition 5.1 Let $M=M\left(e_{0} ; r_{1}, r_{2}, r_{3}\right)$ be a small Seifert manifold with integer Euler class $e_{0}=-2$. If $M$ admits a tight contact structure $\xi$ with $t(\xi)<-1$, then $-M$ admits a positively transverse contact structure. 
Proof Assume that $M$ admits a tight contact structure $\xi$ with $t(\xi)<-1$. Then by Corollary $2.7 t(\xi) \leq-4$. Take numbers $p_{1}, p_{2}, p_{3}$, and $q$ as in Proposition 2.1 with $q \geq 4$ because $t(\xi) \leq-4$. For $i=1,2,3$ we have $p_{i} / q<-r_{i}<\left(p_{i}+2\right) /(q-2)$ because $p_{i} / q$ is negative. Also, $p_{1}+p_{2}+p_{3}=-2 q-1$.

Define $p_{i}^{\prime}=-\left(p_{i}+q\right)$ and $q^{\prime}=q-2$ so that $p_{i}^{\prime} / q^{\prime}=-1-\left(p_{i}+2\right) /(q-2)$, then $p_{i}^{\prime} / q^{\prime}<-1+r_{i}$ and $p_{1}^{\prime}+p_{2}^{\prime}+p_{3}^{\prime}=-q^{\prime}-1$. This implies that $-M$ carries a transverse contact structure because $-M\left(-2 ; r_{1}, r_{2}, r_{3}\right)=M\left(-1,1-r_{1}, 1-r_{2}, 1-r_{3}\right)$.

By using [16, Theorem 1.1] we have the following corollary:

Corollary 5.2 If a small Seifert manifold $M$ with integer Euler class $e_{0} \leq-2$ is an $L$-space, then $t(\xi)=-1$ for any tight contact structure $\xi$ on $M$.

Proof of Theorem 1.3 The part of the statement concerning the maximal twisted number is Corollary 5.2. It implies, by Proposition 2.1 and Proposition 3.3, that the only possibility for the background of $\xi$ is $\beta(-1,-1,1)$. After some easy arithmetics, from the classification of tight contact structures on solid tori [11, Theorem 2.3] we have $T\left(r_{1}\right), T\left(r_{2}\right)$, and $T\left(r_{3}\right)$ possible isotopy classes of tight contact structures on the neighbourhoods of the singular fibres $U_{1}, U_{2}$, and $U_{3}$ respectively. Altogether, they give an upper bound of at most $T\left(r_{1}\right) T\left(r_{2}\right) T\left(r_{3}\right)$ isotopy classes of tight contact structures on $M\left(-2 ; r_{1}, r_{2}, r_{3}\right)$.

In order to construct $T\left(r_{1}\right) T\left(r_{2}\right) T\left(r_{3}\right)$ nonisotopic tight contact structures we perform Legendrian surgery on all possible Legendrian realisations of the link in Figure 2 with the appropriate Thurston-Bennequin number on each component. We have a unique possibility for the central unknot with surgery coefficient -2 : it must be a Legendrian unknot with Thurston-Bennequin number -1. An unknot in one of the "legs" with surgery coefficient $a_{j}^{(i)}$ must be made Legendrian with Thurston-Bennequin number $a_{j}^{(i)}+1$, therefore we have $\left|a_{j}^{(i)}+1\right|$ choices for its rotation number. Varying over all possible choices of the rotation number for all components of the links produces $T\left(r_{1}\right) T\left(r_{2}\right) T\left(r_{3}\right)$ Stein fillable contact structures whose fillings are all diffeomorphic, but have pairwise distinct first Chern classes. By [18, Theorem 2] all the contact structures constructed from these surgeries have pairwise distinct and nontrivial OzsváthSzabó invariants with coefficients in $\mathbb{Z} / 2 \mathbb{Z}$, therefore they are pairwise nonisotopic (see also Lisca and Matić [14] for a similar way to prove the same result). Since $M$ is an $L$-space by [16, Theorem 1.1], for any $\operatorname{Spin}^{c}$-structure $\mathfrak{s}$ there is only one nonzero element in $\widehat{H F}(-M, \mathfrak{s})$ with $\mathbb{Z} / 2 \mathbb{Z}$ coefficients. This implies that the tight contact structures defined by all possible Legendrian surgeries on the link in Figure 2 define pairwise distinct $\operatorname{Spin}^{c}$-structures. 


\section{References}

[1] J B Etnyre, Legendrian and transversal knots, from: "Handbook of knot theory", Elsevier B. V., Amsterdam (2005) 105-185 MR2179261

[2] J B Etnyre, K Honda, Knots and contact geometry. I. Torus knots and the figure eight knot, J. Symplectic Geom. 1 (2001) 63-120 MR1959579

[3] D Gay, A Stipsicz, Symplectic rational blow-down along Seifert fibered 3-manifolds arXiv:math/0703370

[4] P Ghiggini, Tight contact structures on Seifert manifolds over $T^{2}$ with one singular fibre, Algebr. Geom. Topol. 5 (2005) 785-833 MR2153106

[5] P Ghiggini, P Lisca, A I Stipsicz, Classification of tight contact structures on small Seifert 3-manifolds with $e_{0} \geq 0$, Proc. Amer. Math. Soc. 134 (2006) 909-916 MR2180909

[6] P Ghiggini, P Lisca, A I Stipsicz, Tight contact structures on some small Seifert fibered 3-manifolds, Amer. J. Math. 129 (2007) 1403-1447 MR2354324

[7] E Giroux, Convexité en topologie de contact, Comment. Math. Helv. 66 (1991) 637-677 MR1129802

[8] E Giroux, Structures de contact en dimension trois et bifurcations des feuilletages de surfaces, Invent. Math. 141 (2000) 615-689 MR1779622

[9] E Giroux, Structures de contact sur les variétés fibrées en cercles audessus d'une surface, Comment. Math. Helv. 76 (2001) 218-262 MR1839346

[10] E Giroux, Sur les transformations de contact au-dessus des surfaces, from: "Essays on geometry and related topics, Vol. 1, 2", (É Ghys, P de la Harpe, V F R Jones, V Sergiescu, T Tsuboi, editors), Monogr. Enseign. Math. 38, Enseignement Math., Geneva (2001) 329-350 MR1929332

[11] K Honda, On the classification of tight contact structures. I, Geom. Topol. 4 (2000) 309-368 MR1786111

[12] K Honda, On the classification of tight contact structures. II, J. Differential Geom. 55 (2000) 83-143 MR1849027

[13] K Honda, Gluing tight contact structures, Duke Math. J. 115 (2002) 435-478 MR1940409

[14] P Lisca, G Matić, Stein 4-manifolds with boundary and contact structures, Topology Appl. 88 (1998) 55-66 MR1634563Symplectic, contact and low-dimensional topology (Athens, GA, 1996)

[15] P Lisca, G Matić, Transverse contact structures on Seifert 3-manifolds, Algebr. Geom. Topol. 4 (2004) 1125-1144 MR2113899

[16] P Lisca, A I Stipsicz, Ozsváth-Szabó invariants and tight contact three-manifolds, III arXiv:math.SG/0505493 
[17] P Massot, Geodesible contact structures on 3-manifolds arXiv:0711.0377

[18] O Plamenevskaya, Contact structures with distinct Heegaard Floer invariants, Math. Res. Lett. 11 (2004) 547-561 MR2092907

[19] P Scott, The geometries of 3-manifolds, Bull. London Math. Soc. 15 (1983) 401-487 MR705527

[20] H Wu, Legendrian vertical circles in small Seifert spaces, Commun. Contemp. Math. 8 (2006) 219-246 MR2219613

Mathematics 280-37, California Institute of Technology

Pasadena, CA 91125

ghiggini@caltech.edu

Received: 10 October 2007

Algebraic 83 Geometric Topology, Volume 8 (2008) 